\title{
Experiments for Educating Electromagnetic Effects
}

\author{
Frank Leferink ${ }^{(1,3)}$, Istvan Knijff ${ }^{(1,2)}$, Anne Roc' $^{(1)}$ \\ ${ }^{(1)}$ University of Twente, Enschede, The Netherlands \\ ${ }^{(2)}$ Sensata, Almelo, The Netherlands \\ ${ }^{(3)}$ THALES, Hengelo, The Netherlands \\ Frank.Leferink@UTwente.nl
}

\begin{abstract}
Electromagnetic fields are considered by many students as a difficult subject. Unwanted electromagnetic fields are tougher to study. We have developed many experiments as demonstrations (demos) to show the effect of electromagnetic fields in real life products. This paper gives an overview of these demos.
\end{abstract}

\section{INTRODUCTION}

Demos are often used in electromagnetic (field) courses. We have developed many types of demos and used also demos developed by others, such as those described in the IEEE EMC Education Manual [1], or the demo developed within the ASEAN-EU University Network Program [2], or within PATO [3]. We had two main drawbacks: we could easily show the fundamental aspects such as Lenz Law, or crosstalk, in an idealized world, but this was not taken for granted by practicing engineers: these engineers needed a link to their own world. This world was often a printed circuit board. The other drawback was that huge size of many of the demos. Transportation of such demos is therefore a problem.

The demos which have been developed are sized 100x160mm printed circuit boards (PCB). Test equipment consists of a basic dual channel oscilloscope, and, if available, a basic spectrum analyzer.

The detailed description of the demo PCBs are available on a website, for free.

\section{DESCRIPTION OF DEMO PCBS}

A picture of the kit is shown in Figure 1.

This kit contains demos for showing the effect of:

- Lenz 1 - foldable

- Lenz 2 - fixed loops

- Proximity

- Ground Slot 1 - active

- Ground Slot 2 - passive

- Via Bypass

- Symmetry - signal

- Symmetry - power

- Inductance of Capacitors

- Grounding or floating heat sink

- Ground Bounce - DIL vs. SMD

- Ground Bounce - power layout
A short description of every demo is available, giving:

- Description

- Expected result

- Practical result

- Explanation

- Lesson learned

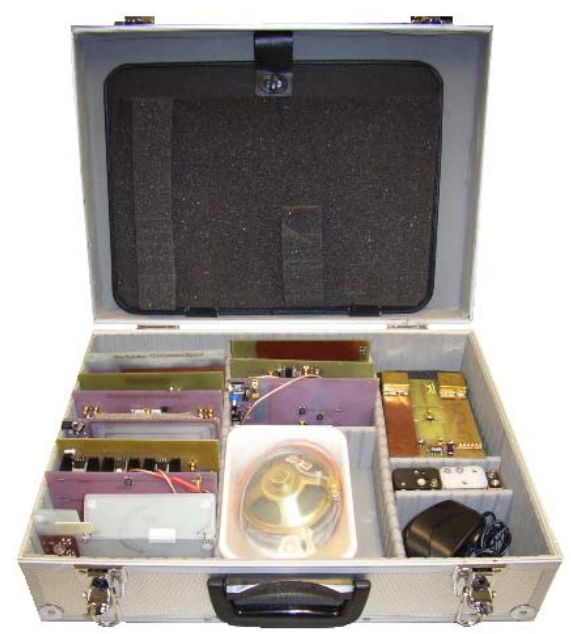

Fig 1: The demo PCB kit

The demos are also used in a practical training, and then students have to measure the effects, give an explanation themselves and draw conclusions from the experiments. We use different questionnaires for this hands-on training.

Some demos are described in detail in this paper. They are entitled: "Lenz, fixed loops", "Inductance of capacitors", "ground bounce" and "grounding and floating heatsink".

A. Lenz, fixed loops.

\section{1) Description}

Four signal tracks with the same load, but different lay-out and width. The Loop4 is the widest, and thus has the lowest resistance. (But it is the largest loop, and thus high inductance.......) 


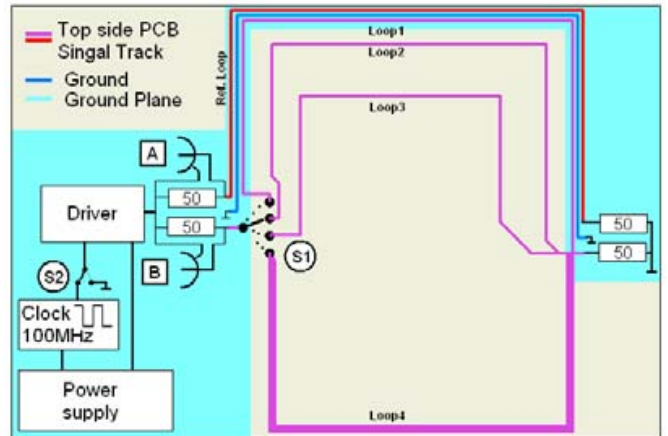

Fig. 2: The Lenz' Law experiment

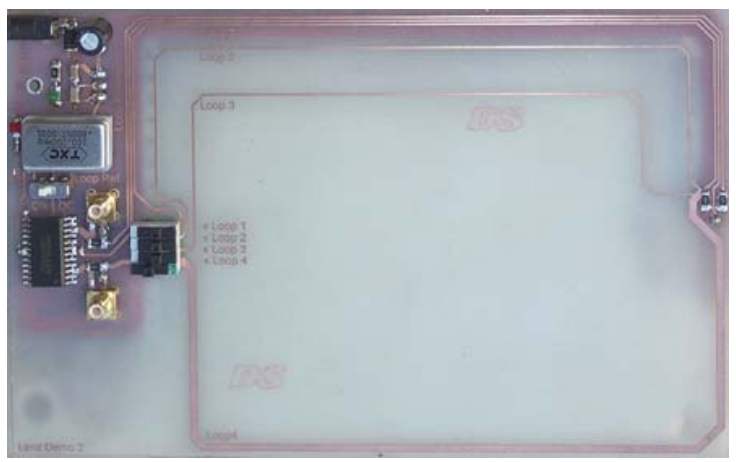

Fig. 3: The Lenz' Law experiment, the PCB

2) Expected result (students)

The signal travels best via the lowest resistance path.

\section{3) Practical result}

The signal is switched between all four tracks. Track 1 matches the original signal best, thus nearly all current flows in the loop with highest resistance (dark blue). The other loop show lower amplitude, thus less current (light blue).

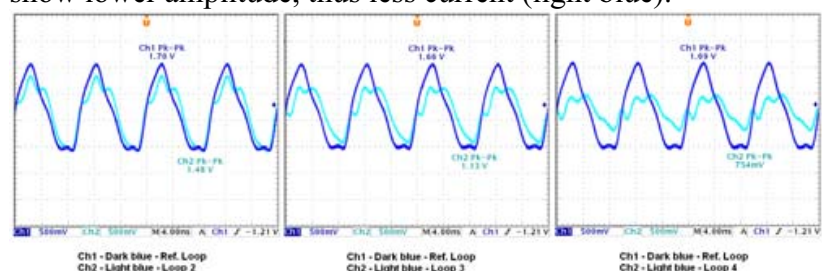

Fig. 4: The Lenz' Law experiment, the measurement result. Left loop 2, then loop 3 and right loop 4 .

\section{4) Explanation}

A return path via the ground plane is available only below signal track 1 (top). Therefore a large current loop is created in the bottom three signal paths. At $100 \mathrm{MHz}$, this is a large inductance which adds to the total path impedance. Actually the narrowest and longest track is best because it has the lowest inductance.

\section{5) Lesson Learned}

Impedance is not only resistive, but also includes inductance. Be aware of loops in your design: keep signal track and ground plane close.

\section{B. Inductance of capacitors}

Non-ideal behavior of passive components.

\section{1) Description}

This board demonstrates the effect of track length, component types and packaging of capacitors. It contains 5 sections:

1. effect of distance between vias and $1 \mathrm{nF}$ capacitors

2. effect of different capacitors: electrolytic, plastic film, ceramic etc.

3. effect of different values of capacitors at high frequencies

4. effect of package of same value capacitors

5. effect of the trace length

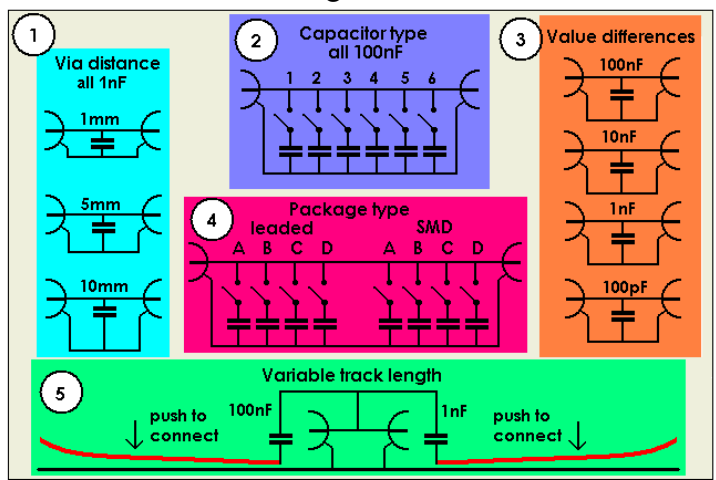

Fig. 5: The passive components experiment

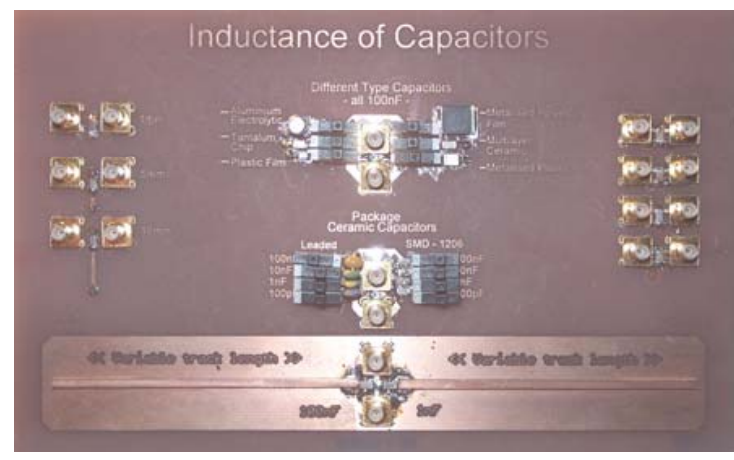

Fig. 6: The passive components experiment, the PCB

\section{2) Expected result}

A component does what the specifications say. The PCB design and lay-out is not included in the circuit diagram.

\section{3) Practical result}

Yes, the components behavior is as expected ... at low frequencies. 
For the first section the influence of the track inductance and the capacitor is clearly demonstrated by the decreasing resonance frequency with increasing track length. Even a small PCB track between the capacitor and the grounding via is used; the component becomes an inductor at appr. $75 \mathrm{MHz}$, as shown in Figure 7.

The section 2 and 3 shows the importance to choose the appropriate capacitor for an application. The impedances of different types of capacitors are clearly different.

In the section 4 the leaded version shows a lower resonance frequencies and higher impedances due to the additional length.

In the section 5, two capacitors in parallel give an antiresonance, such as shown in Figure 8.

\section{4) Explanation}

The actual current loops generate electromagnetic fields which in turn induce unwanted effects.

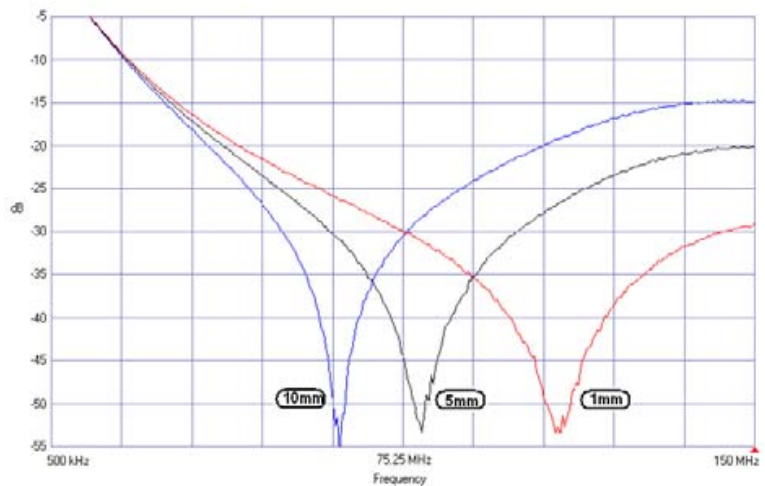

Fig. 7: The passive components experiment, effect of distance between component and via

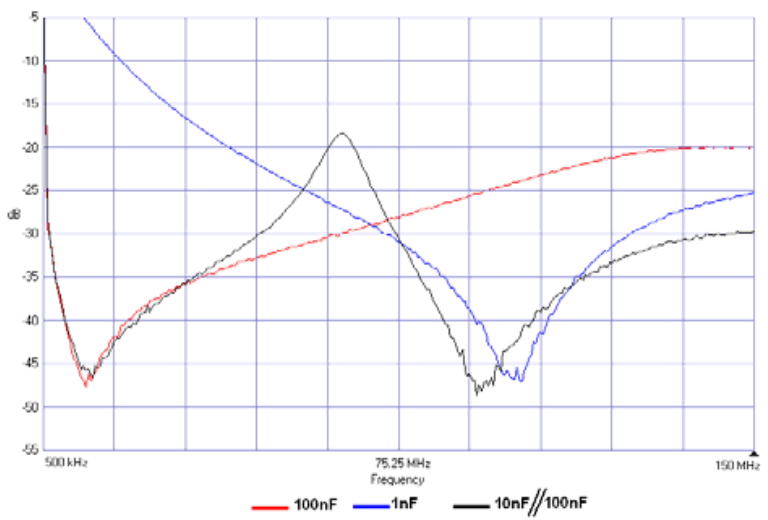

Fig. 8: The passive components experiment, effect of value of capacitor at high frequency, and also two capacitors in parallel

\section{5) Lesson Learned}

The effects of track length, capacitor technology and types of packaging cannot be ignored in the design of high frequency (high-speed) circuits; The behavior of components is not ideal, and even signal track length is an important aspect to consider.

\section{Ground bounce}

\section{1) Description}

Two demos, one with different packages: DIL and SMD. The other with the same package, but with different pins used for power: edge or center pin. The drawing of the latter is shown in Figure 9. The picture of the experiment is presented in Figure 10.

\section{2) Expected result}

No difference in behavior: the components have equal specifications.

\section{3) Practical result}

Two effects are considered:

1. The ground bounce differences between two package types: the DIL package shows much more ground bounce.

2. The ground bounce differences as function as the pin location: it is reduced for outputs pins close to the IC's ground pin as shown in Figure 11.

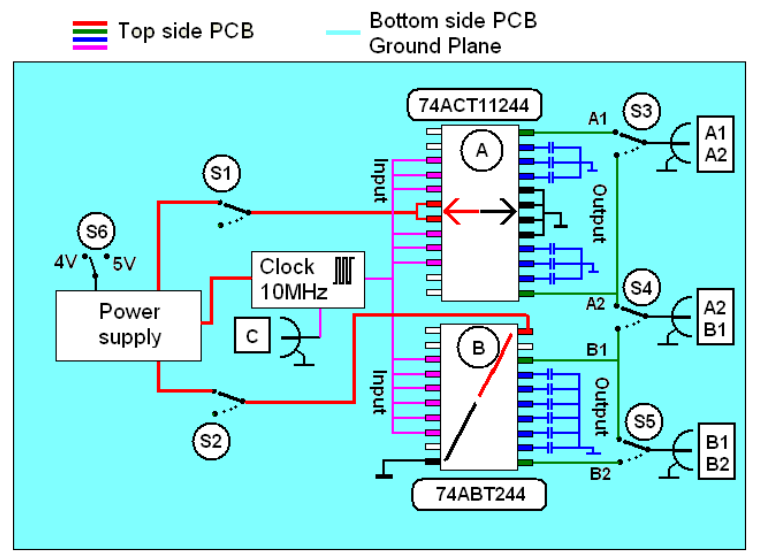

Fig. 9: Ground bounce in a package with edge pin and center pin power supply

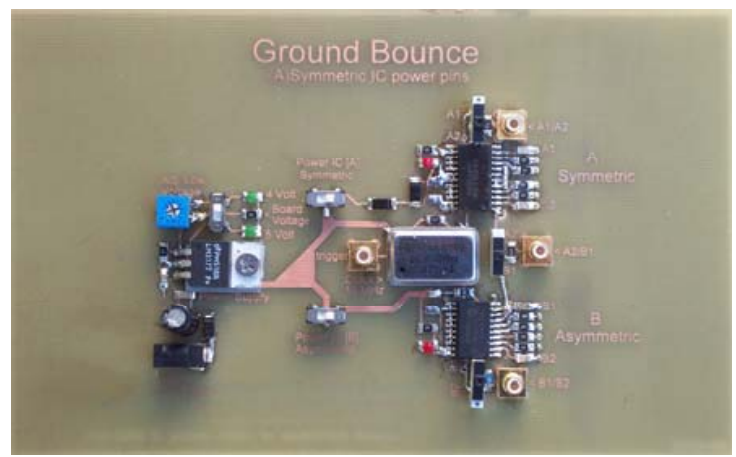

Fig. 10: Ground bounce in a package with edge pin and center pin power supply, the PCB 


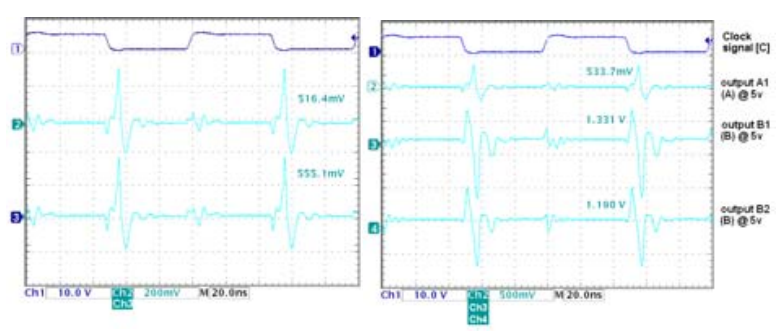

Fig. 11: Ground bounce in a package with edge pin and center pin power supply, measurement results

\section{4) Explanation}

The inductance of the DIL package is higher than the inductance of the SMD package. The ground pin is shared, i.e. a common impedance. Switching current of one output driver will develop a voltage over the inductance. And this voltage is also seen by the non-active driver.

\section{5) Lesson Learned}

The inductance of the package can have a large influence on the behavior of the component.

\section{Grounding or floating heatsink}

\section{1) Description}

The experiment consists of 3 transistors-heatsink configurations. In all cases the heatsink are electrically isolated from the transistor, which have a fall time of $80 \mathrm{~ns}$.

The configuration of the PCB is presented in Figure 12 . The transistor $\mathrm{A}$ is isolated with a conventional silicone pad; the transistor B has an unrealistic distance between transistor and heatsink of $3.2 \mathrm{~mm}$, to demonstrate the capacitive effect. The transistor $\mathrm{C}$ is isolated with two silicon pads with a thin copper sheet between them. This sheet can be connected to ground with a switch. The experiment is presented in Figure 12 and 13.

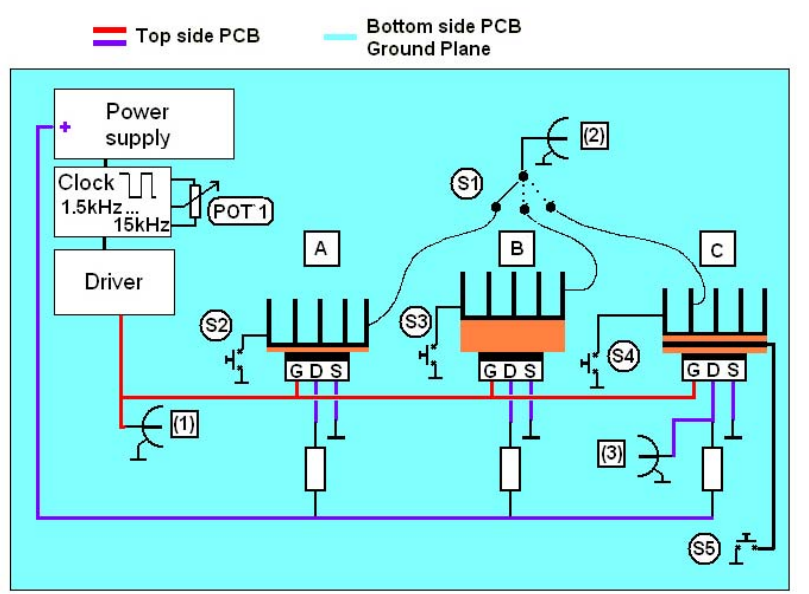

Fig. 12: The heatsink experiment with 3 transistor heatsink configuration

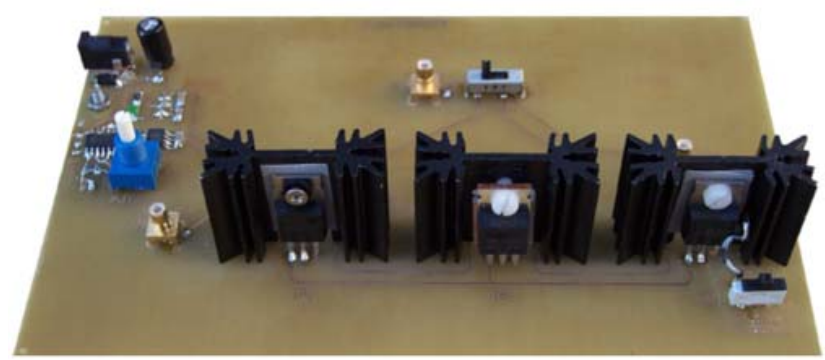

Fig. 13: The heatsink experiment, the PCB

\section{2) Expected result (student)}

The transistor is electrically isolated from the heatsink so no HF noise causing radiated emissions is expected.

\section{3) Practical result}

The capacitive coupling between transistor and heatsink will cause HF noise on heatsink, which will act as an antenna. Connecting the heatsink to ground results in a good noise reduction. The heatsinks then provide a direct return current path for the signal. Ground noise is therefore negligible compared to the floating configuration. The transistor coupled voltages on heatsinks are presented in Figure 13.

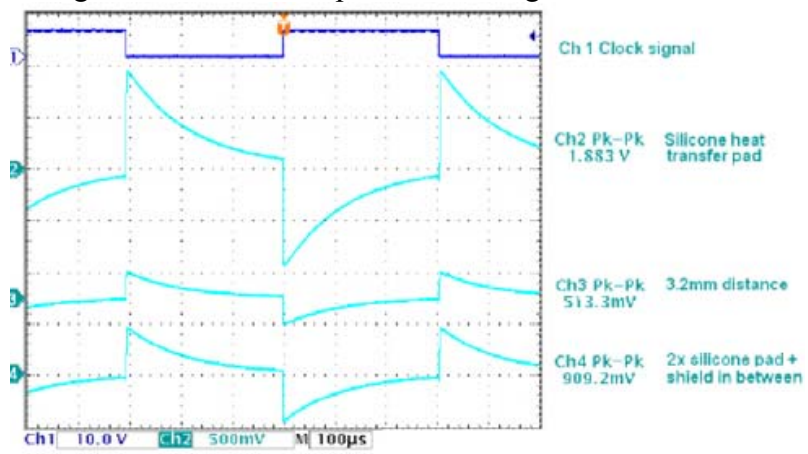

Fig. 13: From transistor coupled voltages on heatsinks

\section{4) Explanation}

The voltage on the transistor couples to the heatsink. Increasing distance is no option due to thermal resistance. A capacitive shield however creates a low inductive return path minimizing the unwanted HF energy in the heatsink.

\section{5) Lesson Learned}

The radiated emission from a heatsink can be minimized by connecting it to the transistors reference voltage. If the reference voltage is hazardous, a conducting sheet between transistor and heatsink can be used.

\section{CONCLUSION}

Demos and experiments are very useful in educating novice engineers, as well as providing practical insight to experienced engineers. It creates an 'aha' effect which cannot be achieved by equations alone. 


\section{REFERENCES}

[1] IEEE EMC Education Manual,

http://www.ewh.ieee.org/soc/emcs/edu/educomms.htm

[2] ASEAN-EU data: http://www.kmitl.ac.th/emc/emitoolkit.htm and http://www.aunp-emctraining.polito.it/index.asp

[3] PATO, Post Academic EMC course, The Netherlands

[4] Istvan Knijff, Design of Electromagnetic Interference Demos, M.Sc.

Thesis, University of Twente, 2005 